\title{
AUTOMATED MONITORING OF CROP TYPE STATISTICS, USING IMAGE CLASSIFICATION
}

\author{
A.Krishna Kumar ${ }^{1}$, D.Deepika ${ }^{2}$ \\ Assistant Professor, ECE Department, Chaitanya Bharathi Institute of Technology, \\ Gandipet, Hyderabad. Telangana ${ }^{1}$ \\ Assistant Professor, CSE Department, Mahatma Gandhi Institute of Technology, Gandipet, \\ Hyderabad. Telangana ${ }^{2}$ \\ a.krishnakumar@yahoo.co.in ${ }^{1}$ \\ deshmukhdeepika@gmail.com ${ }^{2}$
}

DOI: 10.26821/IJSHRE.9.4.2021.9405

\begin{abstract}
Automated monitoring of crop type statistics, is useful in determining the import needs of a country. For instance, if crop area of Onion, is relatively less in a year, then government can plan for importing onions, when onions price is low in the world market. The project aims at analyzing the satellite images, and calculating the crop areas.

The project has three modules: CAI (Crop Area Image) module is used to provide the satellite images, as input to the system. $A D$ (Area determination) module is used to analyze the image, and determine different crop areas. Reporting module is used to generate the crop statistics report.

Image processing technique has been proved as effective machine vision system for agriculture sector. The statistics report can be further visualized in the form of graphs for effective interpretation. Thus, this approach helps to save the environment as well as the cost. Thus, we can conclude that image processing as the most efficient and effective tool that can be applied for the agriculture sector with. great accuracy for analysis of agronomic parameters.
\end{abstract}

Keywords - Crop Area Image, Area determination, pixel-based segmentation algorithm, image cascading algorithm

\section{INTRODUCTION}

Automated monitoring of crop type statistics, is useful in determining the import needs of a country. For instance, if crop area of Onion, is relatively less in a year, then government can plan for importing onions, when onions price is low in the world market. The project aims at analyzing the satellite images, and calculating the crop areas. It uses PyQt tool to create the needed Graphical User Interfaces, PyUIC module to automatically generate the automated code and python image Processing library (Pillow).

The project uses the pixel-based segmentation algorithm to calculate the areas of different types of crops, and image cascading algorithm to identify different types of crops. The images of various types of crops are stored in an image file system. The file system will be stored at a specific location in Ubuntu directory structure. The location will be fed as in input to the system through a GUI screen. The GUI screens in the project are created by a python tool called PyQt.

PyQt is a Python binding of the cross-platform GUI toolkit Qt, implemented as a Python plug-in. PyQt is developed by the British firm Riverbank. PyQt supports Microsoft Windows as well as various flavors of UNIX, including Linux and MacOS. PyQt implements different classes and methods including: classes for accessing SQL database Computing (ODBC, MySQL, PostgreSQL, Oracle, SQLite), Scintilla-based rich text editor widget, data aware widgets that are 
Volume 9 Issue 4 April 2021

automatically populated from a database, an XML parser and SVG support. Scalable Vector Graphics (SVG) is an XML-based vector image format for graphics with support for interactivity. All these features of PyQt, are extensively used in the project, to create the needed Graphical User Interfaces. PyUIC tool is used automatically generate the code for the Front-end user interfaces created by PyQt. All the front-end python code is automatically generated by this tool, by converting the user interface(.ui) files into .py files. [2]

\section{DESIGN METHODOLOGY}

An automated tool is developed by using Python along with its layout toolkit PyQt. Various widgets of PyQt are elaborately used in the forms of this tool as needed. This tool is used to design the needed graphical user interfaces for entering BGR code details, file location details, analyze statistics. With the help of this tool we can determine the crop areas from the satellite images. In the automated assistance for crop type statistics we can ascertain the image and the area details. We can also store the test results of the crop statistics in this automated tool. After the identification, it will finally generate a report using the reporting module.

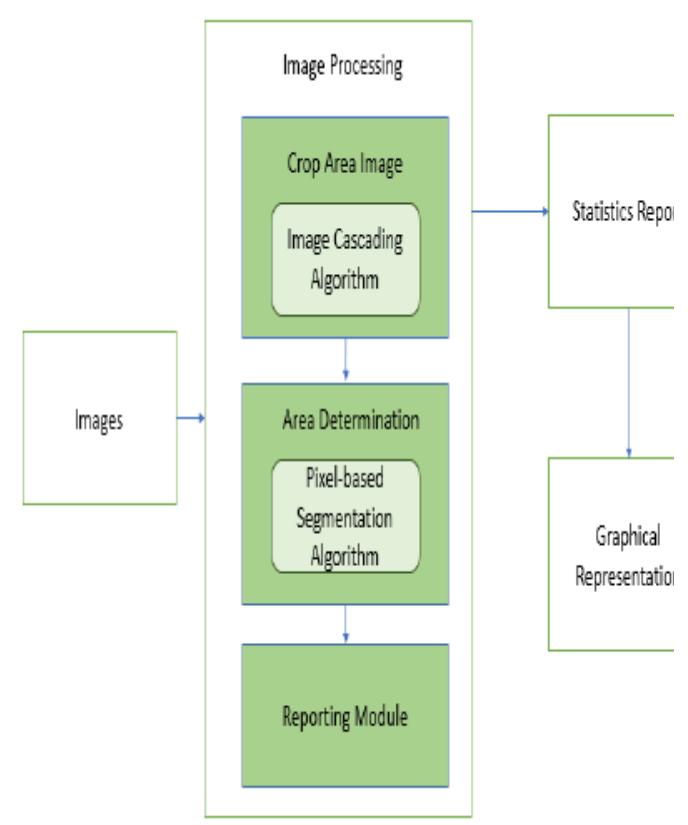

Fig 1 Architecture of Crop type statistics
Fig 1 shows the architecture of the proposed crop type statistics system. The images of various types of crops are stored in an image file system. For processing the image, there are three modules. CAI (Crop Area Image) module is used to cascade the images using the image cascading algorithm. AD (Area determination) module is used to analyze the image by using pixel-based segmentation algorithm and determines different crop areas. Reporting module is used to generate the crop statistics report which can be visualized by graphical representations.

\section{Image Cascading Algorithm}

From the map file

Open a color image and store it in a variable (say, im)

From im

Get colors preset in im

Store the color codes in a variable (say colors)

Initialize max_occurances $=0$, most_present $=0$

For $\mathrm{c}$ in color:

If c[0]>max_occurance:

$($ max_occurance, most_present $)=c$

Print most_present[:3] //Print first 3 values of

most_preference

In case of an exception:

Print "Too many colors in the image"

Repeat for each color image in the map file

\section{Pixel- based segmentation Algorithm}

Open the map file and store it in im 1

Initialize all the color image to 0 and othr $=0$

For pixel in im1.get data ( ): //getdata() will return tuples in RGB format

Pixel 1=pixel [:3] //stores the first pixel parameter in pixel 1

if (pixel 1= most_present [:3] )

$\operatorname{clr} 1+=1$

elif (pixel1 == most_present2[:3]):

$\operatorname{clr} 2+=1$

elif (pixel1 == most_present3[:3]):

$\operatorname{clr} 3+=1$

elif (pixel1 == most_present4[:3]):

clr4 $+=1$

elif (pixel1 == most_present5[:3]):

$\operatorname{clr} 5+=1$

elif (pixel1 == most_present6[:3]):

clr6 += 1

elif (pixel1 == most_present7[:3]):

$\operatorname{clr} 7+=1$

else:

othr $1+=1$

print "Pixel areas of different colors in the map are as follows"

print $" \mathrm{clr} 1=$ ' $\quad+\quad \operatorname{str}(\operatorname{clr} 1)+{ }^{\prime},{ }^{\prime}+\operatorname{cll} 2={ }^{\prime} \quad+$ $\operatorname{str}(\operatorname{clr} 2)++^{\prime},+' \operatorname{clr} 3=$ + $\quad$ str(clr3)+','+'clr4=' + $\operatorname{str}(\operatorname{clr} 4)++^{\prime},+{ }^{\prime} \operatorname{clr} 5='+\operatorname{str}(\operatorname{clr} 5)+{ }^{\prime},{ }^{\prime}+' \operatorname{clr} 6='+$ str(clr6)+','+'clr7=' + str(clr7)+','+'othr1='+str(othr1)" 
Volume 9 Issue 4 April 2021

The project is basically categorized into 3 modules. The three modules are as follows

Crop Area Image (CAI)

Area Determination (AD)

Reporting module

\section{Crop Area Image (CAI):}

Crop area image module is used to provide the inputs to the system, which are the satellite images. After providing inputs to the system, the crop areas are to be calculated. To accomplish second module is used.

\section{Area Determination (AD):}

The second module, that is, area determination module analyzes the satellite images and determines different crop areas. The area can be determined using three measurements, which include pixels, percentages and areas (square kilometers).

\section{Reporting Module:}

Finally, a report is to be created for all the determined crop areas. The third module, that is, the reporting module is used to generate the crop statistics report.

\section{TESTING AND RESULTS}

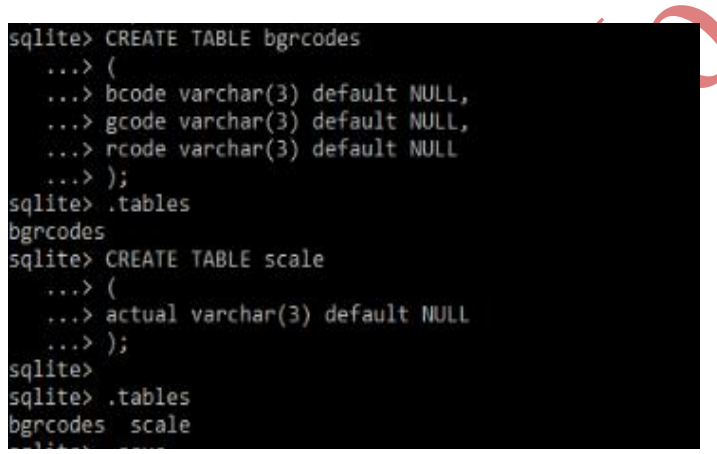

Fig 2 Creation of tables

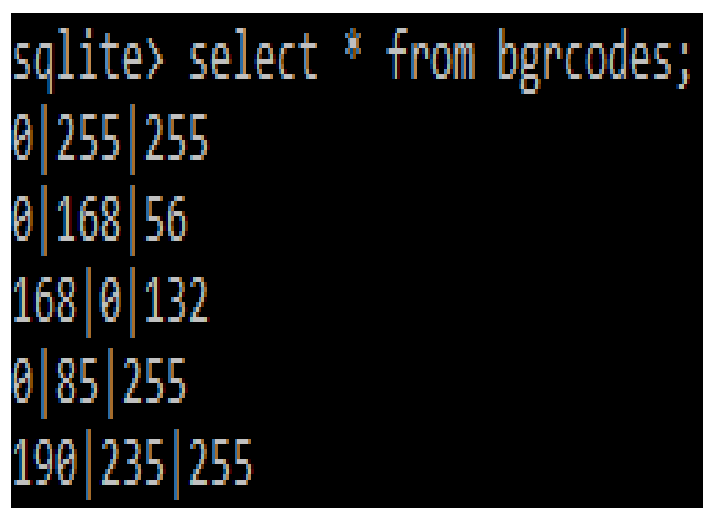

Fig 3 Display of tables
The Fig 2 and Fig 3 displays the information regarding creation of tables. The Fig 2 shows the table creation for bgr codes and scale. The command ".tables" confirms that the table has been created. We can retrieve the created table using select command. Fig 3 shows the retrieval of bgrcodes table.

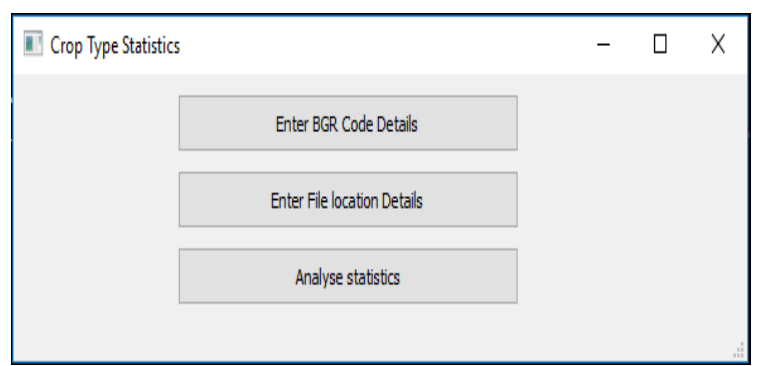

Fig 4 UI for Crop type statistics

The Fig.4 shows the user interface for "crop type statistics" which is created using the pyQT tool. It has three buttons.

- Storage bgr code details

- Enter file location details

Analyse statistics

We can navigate to different user interfaces screens using corresponding buttons.

The bgr code details navigates to BGR codes UI screen, file location details navigate to flocations UI screen and the analyse statistics display the area measurements.
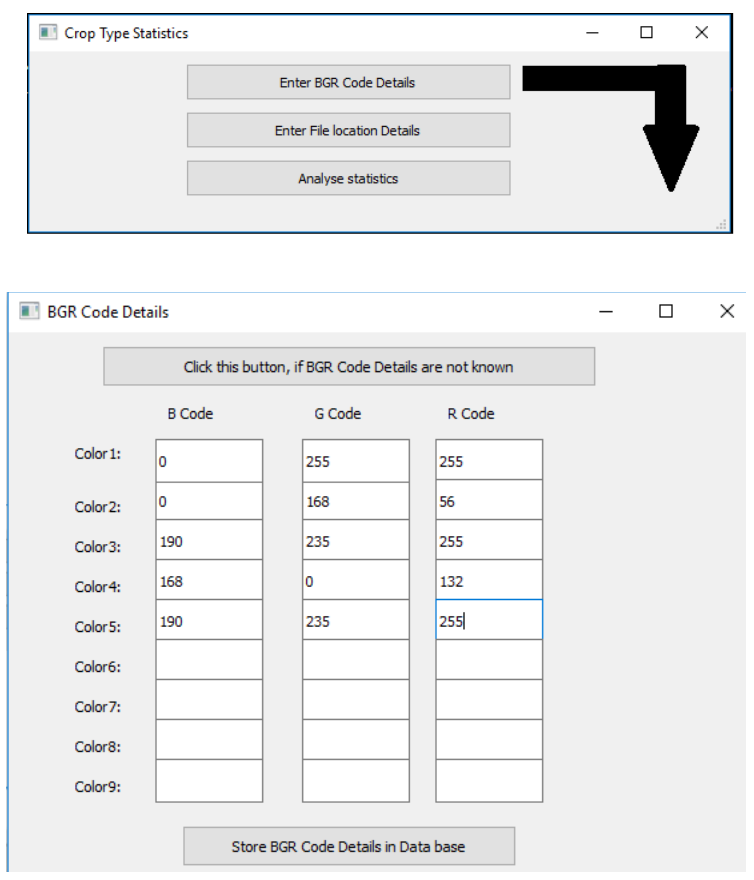

Figure 5 UI for storing color codes (BGR) 
Figure 5 shows the navigation to "BGR code details" UI screen from "crop type statistics" UI screen. The "bgr code details" screen is used to enter the color codes of different images. The button present at the bottom of this screen is "store BGR code details in database". On clicking this button, the entered color codes are stored in the database.
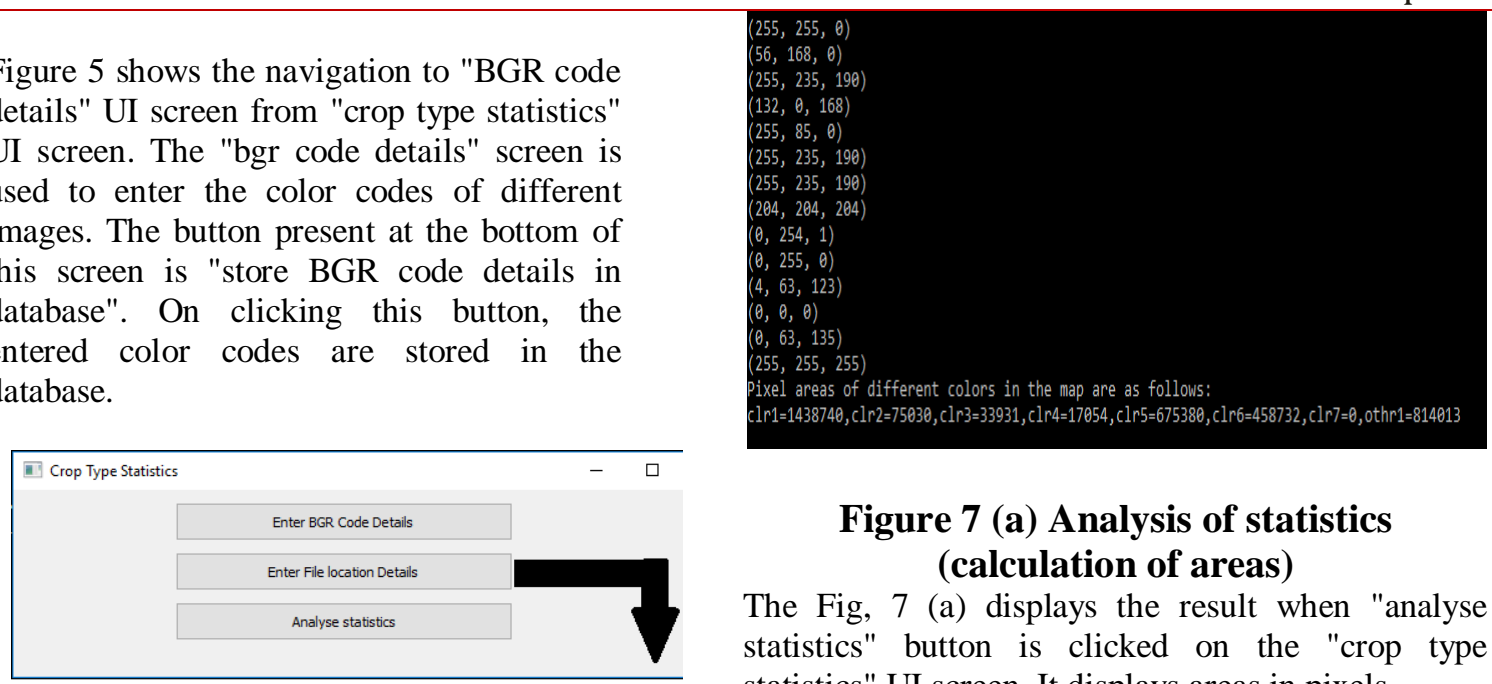

\section{Figure 7 (a) Analysis of statistics (calculation of areas)}

The Fig, 7 (a) displays the result when "analyse statistics" button is clicked on the "crop type statistics" UI screen. It displays areas in pixels .
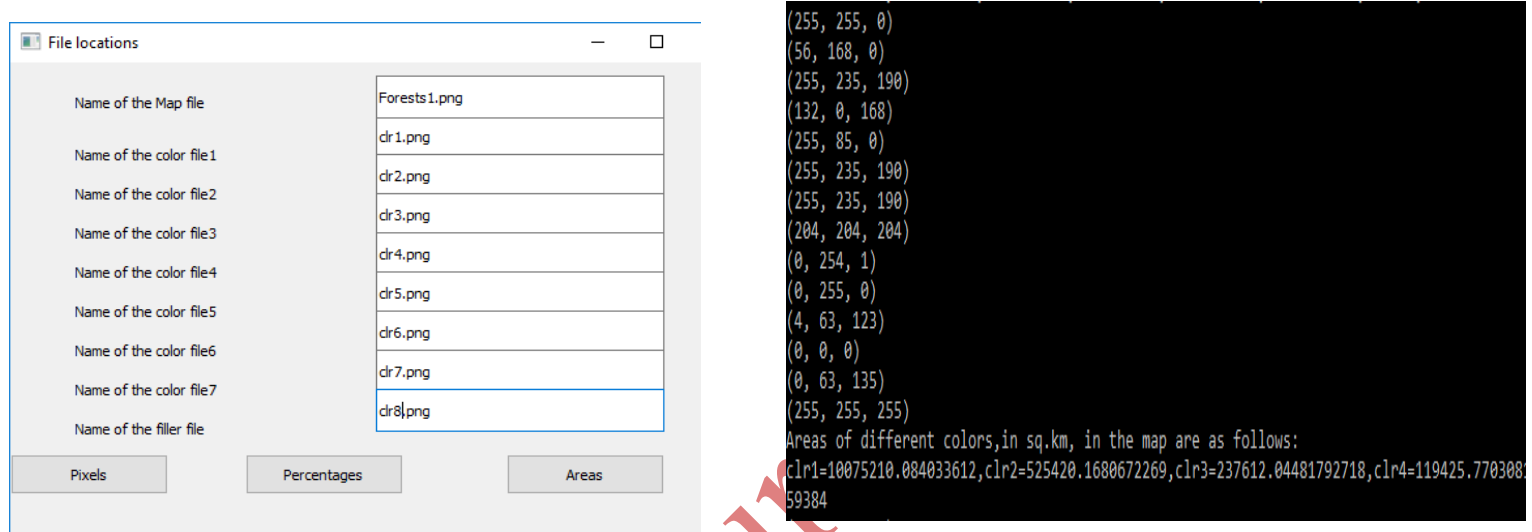

Fig. 6 UI for entering file locations

The Fig. 6 shows the navigation to "file
location" UI screen from "crop type location" UI screen from "crop type
statistics" UI screen. The map file and color file names are entered in this screen. There are 3 buttons at the bottom of the screen which include pixels, percentages and areas. By clicking on "pixels" the areas pf images will be displayed in pixels measurement. Similarly, by clicking on "percentages" or "areas", the image areas will be displayed in percentage or square kilometers measurement respectively.

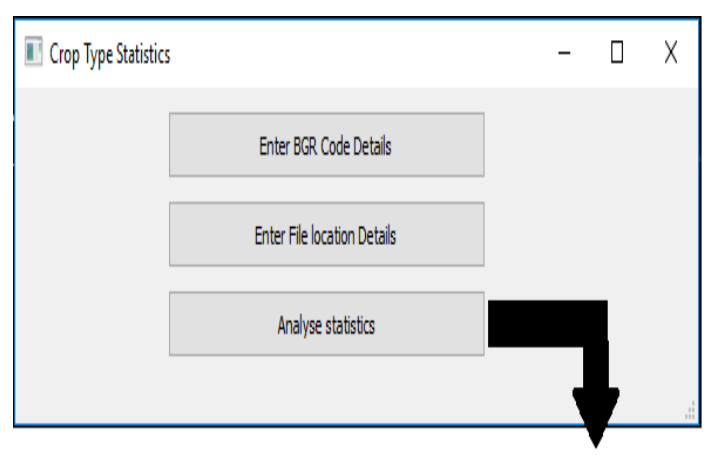

Fig. 7 (b) Analysis of statistics (calculation of areas in sq.km)

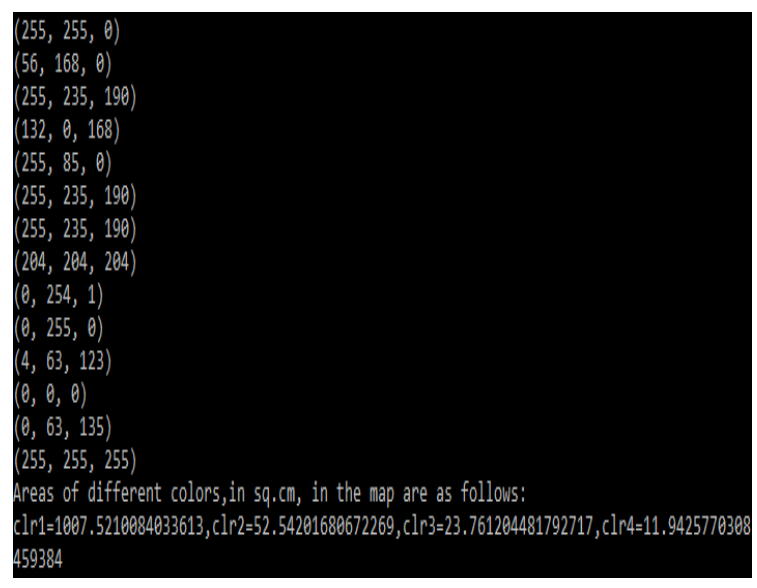

\section{Fig. 7 (c) Analysis of statistics (calculation of} areas in sq.cm)

The Fig, 7 (b) and Fig, 7 (c) display the result when "analyze statistics" button is clicked on the "crop type statistics" UI screen. The areas of different colors in the map are displayed in sq. $\mathrm{km}$ and sq. $\mathrm{cm}$ respectively. 
Volume 9 Issue 4 April 2021

\section{Graphs: \\ Histogram}

In [5]: areadt.areas.plot(kind= "HIST")

Out[5]: <matplotlib.axes._subplots.AxesSubplot at ex1ec2693a898;

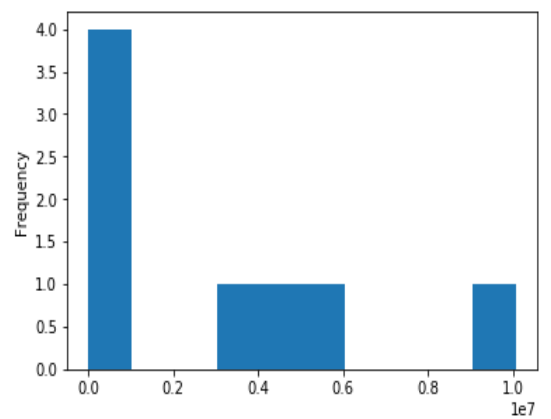

Fig. 8 Histogram

The Fig. 8 is the representation of the histogram graph for the areas calculated. A histogram is a great tool for quickly assessing a probability distribution that is intuitively understood by almost any audience. Python offers a handful of different options for building and plotting histograms. It is an accurate representation of the distribution of numerical data. It is an estimate of the probability distribution of a continuous variable (quantitative variable).

\section{Box Plot}
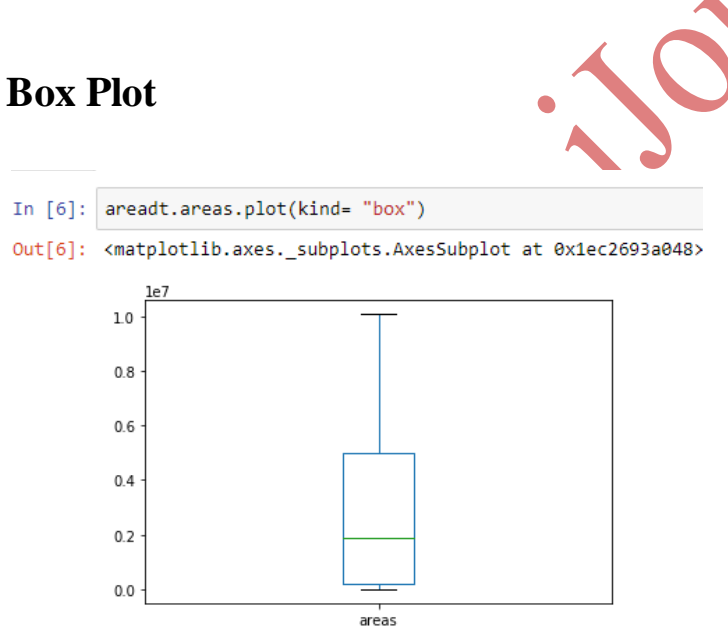

Fig. 9 Box Plot

The Fig. 9 is the representation of the Box plot graph for the areas calculated. A box plot or boxplot is a method for graphically depicting groups of numerical data through their quartiles. Box plots may also have lines extending vertically from the boxes (whiskers) indicating variability outside the upper and lower quartiles, hence the terms box-and-whisker plot and box-and-whisker diagram. Outliers may be plotted as individual points. They display variation in samples of a statistical population without making any assumptions of the underlying statistical distribution.

\section{Density Curve}

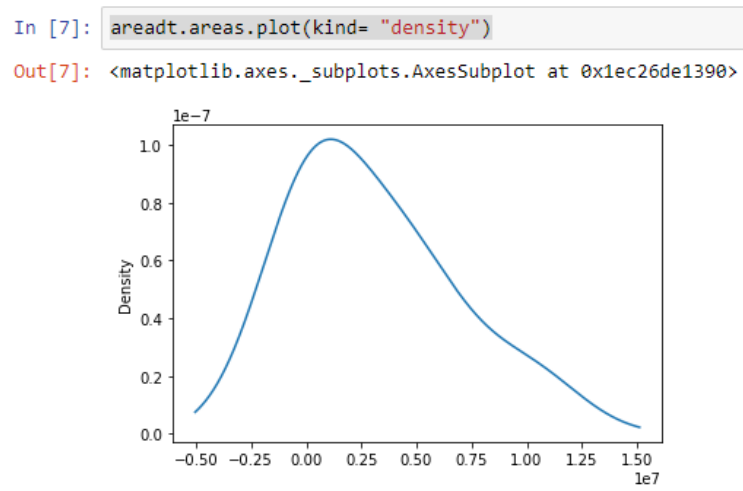

Fig.10 Density Curve

The Fig. 10 is the representation of the Density Curve for the areas calculated. A density plot shows the distribution of a numerical variable. It takes only set of numeric values as input. It is a smoothed, continuøus version of a histogram estimated from the data. The most common form of estimation is known as kernel density estimation. In this method, a continuous curve (the kernel) is drawn at every indiyidual data point and all of these curves are then added together to make a single smooth density estimation.

\section{Pie Chart}

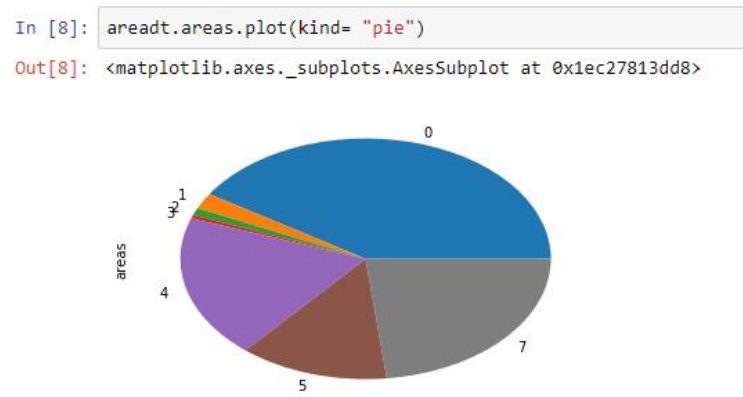

Fig.11 Pie Chart

The Fig. 11 is the representation of the Pie Chart for the areas calculated. A pie chart or a circle chart is a circular statistical graphic, which is divided into slices to illustrate numerical proportion. In a pie chart, the arc length of each slice (and consequently its central angle and area), is proportional to the quantity it represents. While it is named for its resemblance to a pie which has been sliced, there are variations on the way it can be presented. Pie charts are good to show proportional data of different categories. 
Volume 9 Issue 4 April 2021

\section{CONCLUSION AND FUTURE WORK}

\section{Conclusion}

Image processing technique has been proved as effective machine vision system for agriculture sector. The accuracy of classification varies from $85 \%-96 \%$ depending on the algorithms and limitations of image acquisition. Thus, this approach helps to save the environment as well as the cost. Thus, we can conclude that image processing as the most efficient and effective tool that can be applied for the agriculture sector with great accuracy for analysis of agronomic parameters.

\section{Future Work}

The work can be enhanced further by suggestion of crop needs according to the climatic conditions and population for a particular country. It can be further enhanced by allowing monitoring systems to provide continuous data about specific aspects of crop growth and development. Further development of suitable models using artificial intelligence methods such as neural networks and genetic algorithms promise a means of eventually integrating crop data signals on a real-time basis for environmental control. It could be further progressed by allowing the project to suggest which crop would be better for a particular season.

\section{REFERENCES}

[1] "Digital Image Processing applications in agriculture: A Survey" by Asaram Pandurang Janwales in International Journal of advanced research in computer science and software engineering, Volume 5, Issue 3, March 2015 ISSN: 2277128X

[2] "Automated Cropland classification algorithm (ACCA) for California using multi-sensor remote sensing by Zhuoting Wu, Prasad S. Thenkabail, and James P. Verdin in Photogrammetric Engineering and Remote Sensing 80(1):81-90 - January 2014

[3] "Automated classification of landform elements using object-based image analysis" by Lucian Dragul Thomas Blaschke in Volume 81, Issues 3-4, 29 November 2006, Pages 330-344.
[4] "Automated classification of landform, Z. R. B in Iran" by Dr. Maryam Marani Barzanil and Prof. Dr. Khairulmaini Bin Osman Salleh in International Journal of Applied Environmental Sciences ISSN 0973-6077 Volume 11, Number 4 (2016), pp. 915926 ○ Research India Publications

[5] "Digital image processing techniques for detecting, quantifying and classifying plant diseases" by Jayme Garcia Arnal Barbed in Springerplus 2013; 2(1): 660. Published online 2013 Dec 7. doi:10.1186/2193-18012660, PMCID: PMC3863396 PMID:24349961 